\title{
A correlation study of the relationships between nonalcoholic fatty liver disease and serum triglyceride concentration after an oral fat tolerance test
}

Xiaoyu Hou ${ }^{1,2+}$, Yunpeng Guan ${ }^{1,2+}$, Yong Tang ${ }^{2}$, An Song ${ }^{3}$, Jiajun Zhao ${ }^{4}$, Luping Ren², Shuchun Chen², Limin Wei², Huijuan $\mathrm{Ma}^{2}$ and Guangyao Song ${ }^{1,2^{*}}$ D

\begin{abstract}
Background: Nonalcoholic fatty liver disease (NAFLD) has become one of the most common chronic liver diseases worldwide. Triglyceride (TG) accumulation is central to NAFLD development. People now spend most of their day in the postprandial state, and the measurement of postprandial blood lipid concentration can make up for the lack of simple detection of fasting blood lipids. Postprandial triglyceride (PTG) is commonly used as a surrogate for postprandial blood lipid concentrations, and many studies have shown that PTG is a risk factor for NAFLD. The aim of the present study was to investigate the relationship between PTG concentration during oral fat tolerance testing (OFTT) and NAFLD.
\end{abstract}

Methods: A total of 472 Chinese adults, aged 25 to 65 years, were enrolled in the study. All the participants underwent OFTT. The serum concentrations of TG and other lipids were measured, and their relationships with NAFLD were analyzed.

Results: Of the 472 participants, 155 were diagnosed with NAFLD. The fasting and postprandial TG concentrations of the participants with NAFLD were higher than those of healthy participants $(P<0.05)$. The TG concentrations of the healthy participants peaked $4 \mathrm{~h}$ postprandially, whereas those of the participants with NAFLD peaked $6 \mathrm{~h}$ postprandially and reached higher peak values. Postprandial TG concentration was significantly associated with a higher risk of NAFLD.

Conclusions: High PTG is positively related to a higher risk of NAFLD, and the PTG concentrations of patients with NAFLD are higher than in healthy individuals, with a delayed peak. Therefore, 4-h PTG may represent a potential marker of NAFLD.

Trial registration: ChiCTR1800019514.

Keywords: Nonalcoholic fatty liver disease, Postprandial lipidemia, Triglyceride, Oral fat tolerance test, Dyslipidemia, Clinical study

\footnotetext{
* Correspondence: sguangyao2@163.com

${ }^{\dagger}$ Xiaoyu Hou and Yunpeng Guan contributed equally to this work.

${ }^{1}$ Department of Internal Medicine, Hebei Medical University, Shijiazhuang, Hebei, China

2Department of Endocrinology, Hebei General Hospital, 348, Heping West Road, Shijiazhuang, Hebei 050051, People's Republic of China

Full list of author information is available at the end of the article
}

C C The Author(s). 2021 Open Access This article is licensed under a Creative Commons Attribution 4.0 International License, which permits use, sharing, adaptation, distribution and reproduction in any medium or format, as long as you give appropriate credit to the original author(s) and the source, provide a link to the Creative Commons licence, and indicate if changes were made. The images or other third party material in this article are included in the article's Creative Commons licence, unless indicated otherwise in a credit line to the material. If material is not included in the article's Creative Commons licence and your intended use is not permitted by statutory regulation or exceeds the permitted use, you will need to obtain permission directly from the copyright holder. To view a copy of this licence, visit http://creativecommons.org/licenses/by/4.0/ The Creative Commons Public Domain Dedication waiver (http://creativecommons.org/publicdomain/zero/1.0/) applies to the data made available in this article, unless otherwise stated in a credit line to the data. 


\section{Background}

Nonalcoholic fatty liver disease (NAFLD) is the most common liver disease worldwide. It is a clinicopathological syndrome that is characterized by excessive lipid deposition in hepatocytes, and can progress from nonalcoholic fatty liver to nonalcoholic steatohepatitis (NASH) and more severe diseases, which are characterized by hepatocellular injury and fibrosis, including cirrhosis and hepatocellular carcinoma [1, 2]. The proportion of cases of hepatocellular carcinoma that are caused by NAFLD has been increasing rapidly worldwide since 2000, and NAFLD has become the most frequent reason for liver transplantation in the USA [3-5]. Thus, the early identification of people who are more susceptible to NAFLD and the identification of patients in the early stages of NAFLD are of great importance for the prevention and treatment of NAFLD.

The exact mechanism of development of NAFLD is as yet unclear, multiple factors such as insulin resistance, nutritional factors, together with genetic predisposition, have some impacts on the development of NAFLD. In insulin resistant states, the insulin-induced suppression of adipose tissue lipolysis is impaired and the influx of free fatty acids into the liver increases [6]. Triglyceride (TG) is the main form of fat that accumulates in the liver of patients with NAFLD, and the TG content increases alongside lipotoxicity and liver injury. NAFLD is associated with a highly atherogenic lipoprotein profile, characterized by high serum TG, low-density lipoprotein (LDL-C), and apolipoprotein B (ApoB) concentrations and a low HDL-C concentration $[7,8]$. In addition, previous studies have shown that hypertriglyceridemia is one of the risk factors for NAFLD [9], and that the prevalence and degree of hypertriglyceridemia significantly correlate with the severity of NAFLD $[10,11]$.

The fasting lipid profile of patients is most commonly assessed in clinical practice. However, people spend most of their day in the postprandial state TG is mostly affected by diet. Therefore, postprandial triglyceride (PTG) concentration is now commonly used as a surrogate for postprandial lipid status.

Recent studies have shown that dietary composition affects postprandial metabolism [12, 13]. A comparison of the effects of a Western-style high-fat diet, a Western-style high-carbohydrate diet, and a Mediterranean diet on lipid and glucose metabolism showed that energy-rich diets are associated with hyperglycemia, hyperlipidemia, an inflammatory response, and low concentrations of antioxidants [12]. Another study showed that meals that are high in saturated fat and carbohydrate affect the postprandial lipidemia of men with high waist circumference (WC) [13]. However, although various studies have shown a role for postprandial lipidemia, the definition of hypertriglyceridemia varies in different guidelines [14, 15]. A joint consensus statement from the European Atherosclerosis Society (EAS) and European Federation of Clinical Chemistry and Laboratory Medicine stated that the non-fasting TG concentration in patients with a fasting TG $<1.7 \mathrm{mmol} / \mathrm{L}$ should be $<$ $2.0 \mathrm{mmol} / \mathrm{L}(175 \mathrm{mg} / \mathrm{dL})$ [16]. In addition, a Greek expert panel statement defined TG concentrations $\leq 2.5$ $\mathrm{mmol} / \mathrm{L}(220 \mathrm{mg} / \mathrm{dL})$ at all the time-points of an oral fat tolerance test (OFTT) as a desirable postprandial response [17]. Therefore, to further explore the relationship between hypertriglyceridemia and the metabolic abnormalities associated with NAFLD, it is important to establish a standardized OFTT and further define the relationship between PTG concentration and NAFLD.

The aim of the present study was to characterize the relationship between PTG concentration and the incidence of NAFLD by measuring the PTG concentrations during an OFTT, to evaluate the role of postprandial lipidemia in NAFLD.

\section{Methods \\ Study sample}

Four hundred seventy-two volunteers were randomly recruited at the Endocrinology Department of Hebei General Hospital, China, between May 2018 and December 2019. The study complied with the principles of the Declaration of Helsinki, the protocol was approved by the ethics committee of Hebei General Hospital, and all the participants gave their written informed consent. The study has been registered with the China Clinical Trial Registry (registration number: ChiCTR1800019514, registration date: November 15, 2018. http://www.chictr. org.cn/index.aspx).

\section{Exclusion criteria}

Vegetarians; patients with malignant tumors, CVD, diabetes mellitus, thyroid dysfunction, kidney disease, hematologic disease, infectious disease, or psychiatric disorders; individuals who had experienced stroke or had been pregnant in the preceding 3 months; those who were taking drugs that influence lipid metabolism or inflammation, including fish oil, contraceptives, hormones, $\beta$ receptor blockers, or diuretics; and patients who were under antidiabetic therapies and those who had experienced serious infection, surgery, trauma, or a body mass change of $>3 \mathrm{~kg}$ were excluded. After providing their written informed consent, physical examination and oral glucose tolerance testing (OGTT) were performed in all the participants. According to the results of OGTT, diabetic patients were excluded(volunteers whose fasting blood glucose(FBG) $\geq 7.0 \mathrm{mmol} / \mathrm{L}$ with or without 2-h blood glucose $\geq 11.1 \mathrm{mmol} / \mathrm{L}$ were diagnosed as diabetic patients.). 
All the participants also underwent OFTT, as described below.

\section{Oral fat tolerance testing}

All the participants were asked to stick to their normal diet for 1 week before commencing the study. They were studied at Hebei General Hospital after a 10-h overnight fast. Venous blood was collected in the morning, and then the participants consumed a high-fat meal within the following $10 \mathrm{~min}$. The high-fat meal was prepared by professional dieticians, and provided $1500 \mathrm{kcal}$ in total, with fat, protein, and carbohydrate contents of 60,20 , and $20 \%$, respectively. Blood samples were collected $2 \mathrm{~h}, 4 \mathrm{~h}, 6 \mathrm{~h}, 8 \mathrm{~h}$, and $10 \mathrm{~h}$ after the consumption of the high-fat meal and the collected serum samples were stored at $-80^{\circ} \mathrm{C}$ (Haier MDR-382E, China). During this 10-h period, the participants were allowed to drink only water and prohibited from smoking, or eating. Strenuous exercise was also prohibited; only slow walking was permitted.

\section{Measurement of anthropometric, clinical, and biochemical parameters}

The FBG, apolipoprotein A1 (ApoA1), ApoB concentrations, and the total cholesterol (TC), TG, HDL-C, and LDL-C concentrations in the fasting state and $2 \mathrm{~h}, 4 \mathrm{~h}, 6$ $\mathrm{h}, 8 \mathrm{~h}$, and $10 \mathrm{~h}$ after the consumption of a high-fat meal were measured by laboratory technicians in the Physical Examination Center of Hebei General hospital using a Hitachi 7600 automatic biochemical analyzer (Hitachi Instruments Ltd., Tokyo, Japan). Fasting plasma insulin (FINS) concentration was measured using a chemiluminescence method in the Nuclear Medicine Department of Hebei General hospital. Homeostasis model assessment of insulin resistance (HOMA-IR) was calculated using the FINS and FBG concentrations by dividing insulin $(\mu \mathrm{U} / \mathrm{ml})$ and glucose $(\mu \mathrm{mol} / \mathrm{L})$ by $22.5[18]$ $($ HOMA-IR $=$ FBG $\quad((\mathrm{mmol} / \mathrm{L}) \times$ FINS $\quad(\mathrm{mIU} / \mathrm{L}) / 22.5)$. Body mass index (BMI) was calculated as body mass divided by height squared $\left(\mathrm{kg} / \mathrm{m}^{2}\right)$.

Body mass, height, WC, systolic blood pressure (SBP), and diastolic blood pressure (DBP) were measured by trained professionals.

\section{Diagnosis of nonalcoholic fatty liver disease}

Abdominal ultrasonography was performed by a specialist technologist in the Physical Examination Center of Hebei General hospital. A diagnosis of NAFLD was made according to The Guidelines for the Prevention and Treatment of Nonalcoholic Fatty Liver Disease (2018 update) [19] and the position statement on NAFL D/NASH that was derived from the European Association for the Study of the Liver 2009 special conference [20]. NAFLD can be diagnosed by imaging or histological findings of fatty liver, with the exclusion of other causes of liver steatosis (for example, viral, autoimmune, genetic, and use of drugs) and alcohol abuse (consumption of $>30 \mathrm{~g} /$ day in men and $>20 \mathrm{~g} /$ day in women) [20].

Ultrasonographic examinations were performed by a specialist technologist. Diffuse fatty liver was diagnosed if two of the following were present: (1) diffuse enhancement of the near-field echo of the liver ("bright liver") and far-field echo attenuation; (2) unclear intrahepatic duct structure; (3) gradual attenuation of the far-field echo; and (4) mild-to-moderate hepatomegaly with a blunt leading edge.

\section{Statistical analysis}

SPSS 21.0 software (IBM Inc., Armonk, NY, USA) was used for the statistical analysis. Numerical data were tested for normality using the Shapiro-Wilk test. Normally distributed data are expressed as mean \pm standard deviation and non-normally distributed data are expressed as median and interquartile range. The independent sample $t$ test was used to compare data between two groups. Pearson's chi-square test was used to compare the prevalence of NAFLD among the groups. Single-factor ANOVA was used to compare data among three groups. Binary logistic regression analysis, with NAFLD as the dependent variable, was used to determine the influence of each parameter on the prevalence of NAFLD. Statistical significance was accepted when $P<0.05$.

\section{Results}

All the participants consumed the high-fat meal during the OFTT and it was well tolerated.

\section{Basic characteristics}

There were 472 participants in the present study, of whom 224 were male and 248 were female. The mean age of the men was $45 \pm 13$ years and that of the women was $44 \pm 13$ years (Table 1 ).

OGTT was used to exclude diabetic patients (Table S1).

The participants were allocated to the two groups according to their hepatic ultrasonographic findings. The fasting BMI, WC, SBP, DBP, FBG, FINS, TC, TG, LDL$\mathrm{C}, \mathrm{ApoB}$, and HOMA-IR of the NAFLD group were higher than those of the control group (all $P<0.001$ ). However, the HDL-C and ApoA1 concentrations and the ApoA1/ApoB ratio of the NAFLD group were lower than those of the control group $(P<0.001, P=0.001$, and $P<0.001$, respectively) (Table 1 ).

The differences in the fasting BMI, FBG, FINS, HOMAIR, TC, TG, HDL-C, LDL-C, ApoA1, ApoB, and ApoA1/ $A p o B$ of the NAFLD and control groups were affected by sex. The fasting BMI, FBG, FINS, and HOMA-IR of the male and female participants with NAFLD were higher than those of the same sex in the control group $(P<0.001)$. In addition, the fasting TC, TG, and LDL-C concentrations 
Table 1 Basic characters of the participants

\begin{tabular}{|c|c|c|c|c|}
\hline Group & Total $(n=472)$ & Con $(n=317)$ & NAFLD $(n=155)$ & $P$ \\
\hline Age (Year) & $44 \pm 13$ & $43 \pm 14$ & $47 \pm 11^{\# \#}$ & $<0.001$ \\
\hline Sex (Male/Female) & $224 / 248$ & $139 / 178$ & $85 / 70$ & 0.992 \\
\hline $\mathrm{BMI}\left(\mathrm{kg} / \mathrm{m}^{2}\right)$ & $25.93 \pm 4.05$ & $24.45 \pm 3.25$ & $28.97 \pm 3.83^{\# \#}$ & $<0.001$ \\
\hline $\mathrm{SBP}(\mathrm{mmHg})$ & $127 \pm 15$ & $124 \pm 15$ & $133 \pm 14^{\# \#}$ & $<0.001$ \\
\hline $\mathrm{DBP}(\mathrm{mmHg})$ & $78 \pm 10$ & $77 \pm 9$ & $83 \pm 10^{\# \#}$ & $<0.001$ \\
\hline$W C(\mathrm{~cm})$ & $87.9 \pm 11.9$ & $83.9 \pm 10.9$ & $96.1 \pm 9.4^{\# \#}$ & $<0.001$ \\
\hline FBG (mmol/L) & $5.41(5.07,5.87)$ & $5.29(4.97,5.61)$ & $5.78(5.38,6.46){ }^{\# \#}$ & $<0.001$ \\
\hline FINS (mmol/L) & $10.65(7.35,15.24)$ & $8.89(6.43,11.84)$ & $16.15(11.19,19.90) \#$ & $<0.001$ \\
\hline HOMA-IR & $2.60(1.70,3.82)$ & $2.09(1.47,2.95)$ & $4.12(3.01,5.71) \# \#$ & $<0.001$ \\
\hline $\mathrm{TC}(\mathrm{mmol} / \mathrm{L})$ & $4.72 \pm 1.01$ & $4.59 \pm 0.96$ & $5.00 \pm 1.06^{\# \#}$ & $<0.001$ \\
\hline TG $(\mathrm{mmol} / \mathrm{L})$ & $1.66 \pm 1.31$ & $1.34 \pm 1.07$ & $2.32 \pm 1.50^{\# \#}$ & $<0.001$ \\
\hline $\mathrm{HDL}-\mathrm{C}(\mathrm{mmol} / \mathrm{L})$ & $1.25 \pm 0.28$ & $1.30 \pm 0.29$ & $1.14 \pm 0.22^{\# \#}$ & $<0.001$ \\
\hline $\mathrm{LDL}-\mathrm{C}(\mathrm{mmol} / \mathrm{L})$ & $2.98 \pm 0.73$ & $2.85 \pm 0.69$ & $3.23 \pm 0.74^{\# \#}$ & $<0.001$ \\
\hline $\operatorname{ApoA1}(g / L)$ & $1.40(1.24,1.56)$ & $1.42(1.27,1.60)$ & $1.34(1.20,1.51){ }^{\#}$ & $=0.001$ \\
\hline $\mathrm{ApoB}(\mathrm{g} / \mathrm{L})$ & $0.79 \pm 0.22$ & $0.75 \pm 0.20$ & $0.88 \pm 0.23^{\# \#}$ & $<0.001$ \\
\hline ApoA1/ApoB & $1.78(1.48,2.22)$ & $1.94(1.61,2.36)$ & $1.57(1.28,1.86){ }^{\# \#}$ & $<0.001$ \\
\hline
\end{tabular}

Data are mean \pm standard deviation or median (interquartile range)

Abbreviations: BMI body mass index, WC waist circumference, SBP systolic blood pressure, DBP diastolic blood pressure, FBG fasting blood glucose, FINS fasting plasma insulin concentration, HOMA-IR homeostasis model assessment of insulin resistance, $T C$ total cholesterol, $T G$ triglyceride, $H D L-C$ high-density lipoproteincholesterol, LDL-C low-density lipoprotein-cholesterol, ApoA1 apolipoprotein A1, ApoB apolipoprotein B

${ }^{\#} P<0.05$, compared with the Con group. ${ }^{\# \#} P<0.01$, compared with the Con group

of the NAFLD group were higher than those of the control group among participants of the same sex, but the HDL-C concentration was lower than that of the control group (all $P<0.05$ ) (Table 2).

\section{Circulating lipid concentrations at time points during the OFTT}

The TC, TG, and LDL-C concentrations in the fasting state, and $2 \mathrm{~h}, 4 \mathrm{~h}, 6 \mathrm{~h}, 8 \mathrm{~h}$, and $10 \mathrm{~h}$ after the start of the
OFTT were higher in the NAFLD group than in the control group, whereas the HDL-C concentration was lower $(P<0.001)$. Because lipid metabolism is known to differ according to sex, the circulating lipid concentrations at time points during the OFTT were further analyzed. Among the male participants, patients with NAFL D had higher concentrations of TC, TG, and LDL-C, and a lower HDL-C concentration than those of the control group. The TC concentrations in the fasting

Table 2 Comparison of anthropometric, clinical, and fasting metabolic parameters in participants of each sex in the NAFLD and control groups

\begin{tabular}{|c|c|c|c|c|}
\hline & \multicolumn{2}{|l|}{ Con } & \multicolumn{2}{|l|}{ NAFLD } \\
\hline & $\operatorname{Male}(n=139)$ & Female $(n=178)$ & $\operatorname{Male}(n=85)$ & Female $(n=70)$ \\
\hline Age (Year) & $45 \pm 14$ & $42 \pm 13$ & $44 \pm 12$ & $51 \pm 10$ \\
\hline $\mathrm{BMI}\left(\mathrm{kg} / \mathrm{m}^{2}\right)$ & $25.57 \pm 3.17$ & $23.58 \pm 3.04$ & $29.29 \pm 3.55^{\# \#}$ & $28.58 \pm 4.14^{\# \#}$ \\
\hline FBG (mmol/L) & $5.40(5.07,5.79)$ & $5.22(4.93,5.54)$ & $5.79(5.40,6.47) \#$ & $5.78(5.37,6.49) \#$ \\
\hline FINS (mmol/L) & $9.40(6.48,12.34)$ & $8.63(6.14,11.60)$ & $16.40(11.17,20.57) \# \#$ & $15.33(11.45,19.34) \#$ \\
\hline HOMA-IR & $2.09(1.56,3.07)$ & $2.07(1.39,2.76)$ & $4.28(3.04,5.86))^{\# \#}$ & $4.07(2.97,5.67){ }^{\# \#}$ \\
\hline $\mathrm{TC}(\mathrm{mmol} / \mathrm{L})$ & $4.43 \pm 0.86$ & $4.71 \pm 1.02$ & $4.82 \pm 0.99^{\#}$ & $5.21 \pm 1.10^{\#}$ \\
\hline TG (mmol/L) & $0.99 \pm 0.30$ & $0.86 \pm 0.26$ & $1.57 \pm 0.48^{\# \#}$ & $1.78 \pm 0.77^{\# \#}$ \\
\hline $\mathrm{HDL}-\mathrm{C}(\mathrm{mmol} / \mathrm{L})$ & $1.18 \pm 0.26$ & $1.39 \pm 0.29$ & $1.06 \pm 0.16^{\# \#}$ & $1.24 \pm 0.24^{\# \#}$ \\
\hline LDL-C (mmol/L) & $2.79 \pm 0.60$ & $2.90 \pm 0.76$ & $3.14 \pm 0.71^{\# \#}$ & $3.34 \pm 0.78^{\# \#}$ \\
\hline
\end{tabular}

Data are mean \pm standard deviation or median (interquartile range)

Abbreviations: Con control group, BMI body mass index, FBG fasting blood glucose, FINS fasting plasma insulin concentration, HOMA-IR homeostasis model assessment of insulin resistance, $T C$ total cholesterol, $T G$ triglyceride, $H D L$ - $C$ high-density lipoprotein-cholesterol, $L D L-C$ low-density lipoprotein-cholesterol

${ }^{\#} P<0.05$, compared with the Con group. ${ }^{\# \#} P<0.01$, compared with the Con group 
state, and $2 \mathrm{~h}, 4 \mathrm{~h}, 6 \mathrm{~h}, 8 \mathrm{~h}$, and $10 \mathrm{~h}$ after the high-fat meal were significantly higher than those of the control group $(P<0.05, P<0.05, P<0.001, P<0.001, P<0.001$, and $P<0.001$, respectively). The female patients with NAFLD also had significantly higher TC, TG, and LDL$\mathrm{C}$, and lower HDL-C concentrations (all $P<0.001$ ) than those of the control group (Table 3).

\section{TG peak concentration and timing}

A graph of the TG concentration at time points during the OFTT was constructed and analyzed according to sex. The TG concentration in each group increased gradually after the ingestion of the high-fat meal during the OFTT. Regardless of sex, the PTG of the control group peaked $4 \mathrm{~h}$ postprandially while the TG of the NAFLD group peaked $6 \mathrm{~h}$ postprandially (Fig. 1a-c).

\section{Comparisons of parameters among groups of participants with differing fasting TG concentration}

According to their fasting TG concentration, the participants were allocated to one of two groups: those with a fasting TG concentration $\leq 1.7 \mathrm{mmol} / \mathrm{L}$ were placed in a normal-TG group (NFTG group) and those with TG $>1.7 \mathrm{mmol} / \mathrm{L}$ were placed in a high fasting TG group (HFTG group). The fasting BMI, FBG, FINS, and HOMA-IR of the HFTG group were higher than those of the NFTG group $(P<$ 0.001). The incidence of NAFLD in the participants as a whole was $32.8 \%$. Among participants who had a normal fasting TG, the incidence of NAFLD was $19.9 \%$, whereas it was $60.7 \%$ in the high-TG group. Pearson's chi-square test showed that the incidence of NAFLD between the two groups significantly differed $(P<0.001)$ (Table 4$)$.

Table $\mathbf{3}$ Lipid metabolism parameters during the oral fat tolerance test

\begin{tabular}{|c|c|c|c|c|c|c|c|}
\hline & & $\mathrm{Oh}$ & $2 \mathrm{~h}$ & $4 \mathrm{~h}$ & $6 \mathrm{~h}$ & $8 \mathrm{~h}$ & $10 \mathrm{~h}$ \\
\hline \multirow[t]{4}{*}{ Con } & $\mathrm{TC}$ & $4.59 \pm 0.96$ & $4.56 \pm 0.94$ & $4.55 \pm 0.96$ & $4.70 \pm 0.98$ & $4.75 \pm 0.99$ & $4.70 \pm 0.95$ \\
\hline & $\mathrm{TG}$ & $1.34 \pm 1.07$ & $2.20 \pm 1.27$ & $2.65 \pm 1.75$ & $2.59 \pm 2.03$ & $2.33 \pm 2.04$ & $1.57 \pm 1.62$ \\
\hline & $\mathrm{HDL}-\mathrm{C}$ & $1.30 \pm 0.29$ & $1.30 \pm 0.29$ & $1.24 \pm 0.28$ & $1.24 \pm 0.29$ & $1.29 \pm 0.30$ & $1.28 \pm 0.29$ \\
\hline & LDL-C & $2.85 \pm 0.69$ & $2.78 \pm 0.67$ & $2.72 \pm 0.65$ & $2.80 \pm 0.67$ & $2.84 \pm 0.68$ & $2.88 \pm 0.67$ \\
\hline \multirow[t]{4}{*}{ Con (male) } & $\mathrm{TC}$ & $4.42 \pm 0.86$ & $4.42 \pm 0.87$ & $4.43 \pm 0.89$ & $4.59 \pm 0.89$ & $4.63 \pm 0.88$ & $4.61 \pm 0.90$ \\
\hline & TG & $1.56 \pm 1.22$ & $2.47 \pm 1.45$ & $3.11 \pm 1.98$ & $3.15 \pm 2.30$ & $2.92 \pm 2.41$ & $1.98 \pm 2.06$ \\
\hline & $\mathrm{HDL}-\mathrm{C}$ & $1.18 \pm 0.26$ & $1.19 \pm 0.27$ & $1.13 \pm 0.26$ & $1.14 \pm 0.27$ & $1.17 \pm 0.28$ & $1.17 \pm 0.27$ \\
\hline & LDL-C & $2.79 \pm 0.60$ & $2.73 \pm 0.60$ & $2.68 \pm 0.58$ & $2.76 \pm 0.57$ & $2.80 \pm 0.58$ & $2.86 \pm 0.61$ \\
\hline \multirow[t]{4}{*}{ Con (female) } & $\mathrm{TC}$ & $4.71 \pm 1.02$ & $4.67 \pm 0.97$ & $4.65 \pm 1.00$ & $4.78 \pm 1.05$ & $4.83 \pm 1.06$ & $4.77 \pm 0.98$ \\
\hline & TG & $1.17 \pm 0.90$ & $1.99 \pm 1.06$ & $2.29 \pm 1.44$ & $2.15 \pm 1.67$ & $1.87 \pm 1.55$ & $1.26 \pm 1.08$ \\
\hline & $\mathrm{HDL}-\mathrm{C}$ & $1.39 \pm 0.29$ & $1.39 \pm 0.28$ & $1.32 \pm 0.27$ & $1.33 \pm 0.28$ & $1.38 \pm 0.28$ & $1.37 \pm 0.28$ \\
\hline & LDL-C & $2.90 \pm 0.76$ & $2.83 \pm 0.72$ & $2.76 \pm 0.70$ & $2.83 \pm 0.74$ & $2.88 \pm 0.75$ & $2.89 \pm 0.72$ \\
\hline \multirow[t]{4}{*}{ NAFLD } & $\mathrm{TC}$ & $5.00 \pm 1.06^{\# \#}$ & $4.96 \pm 1.03^{\# \#}$ & $5.05 \pm 1.07^{\# \#}$ & $5.22 \pm 1.09^{\# \#}$ & $5.24 \pm 1.10^{\# \#}$ & $5.21 \pm 1.20^{\# \#}$ \\
\hline & TG & $2.32 \pm 1.50^{\# \#}$ & $3.47 \pm 1.59^{\# \#}$ & $4.32 \pm 2.04^{\# \#}$ & $4.50 \pm 2.54^{\# \#}$ & $4.25 \pm 2.61^{\# \#}$ & $2.96 \pm 2.45^{\# \#}$ \\
\hline & $\mathrm{HDL}-\mathrm{C}$ & $1.14 \pm 0.22^{\# \#}$ & $1.15 \pm 0.22^{\# \#}$ & $1.09 \pm 0.21^{\# \#}$ & $1.07 \pm 0.22^{\# \#}$ & $1.11 \pm 0.22^{\# \#}$ & $1.10 \pm 0.22^{\# \#}$ \\
\hline & LDL-C & $3.23 \pm 0.74^{\# \#}$ & $3.14 \pm 0.72^{\# \#}$ & $3.09 \pm 0.70^{\# \#}$ & $3.16 \pm 0.69^{\# \#}$ & $3.20 \pm 0.70^{\# \#}$ & $3.28 \pm 0.75^{\# \#}$ \\
\hline \multirow{4}{*}{$\begin{array}{l}\text { NAFLD } \\
\text { (male) }\end{array}$} & TC & $4.82 \pm 0.99^{\&}$ & $4.78 \pm 0.94^{\&}$ & $4.91 \pm 1.03^{\& \&}$ & $5.08 \pm 1.05^{\& \&}$ & $5.10 \pm 1.07^{\&}$ & $5.08 \pm 1.20^{\&}$ \\
\hline & TG & $2.54 \pm 1.73^{\& \&}$ & $3.73 \pm 1.67^{\& \&}$ & $4.60 \pm 2.07^{\& \&}$ & $4.81 \pm 2.50^{\& \&}$ & $4.52 \pm 2.63^{\& \&}$ & $3.07 \pm 2.45^{\& \&}$ \\
\hline & $\mathrm{HDL}-\mathrm{C}$ & $1.06 \pm 0.16^{8 \&}$ & $1.07 \pm 0.17^{\& \&}$ & $1.02 \pm 0.17^{\&}$ & $1.01 \pm 0.16^{8 \&}$ & $1.04 \pm 0.18^{\& \&}$ & $1.03 \pm 0.17^{\& \&}$ \\
\hline & LDL-C & $3.14 \pm 0.71^{\& \&}$ & $3.05 \pm 0.69^{\& \&}$ & $3.02 \pm 0.68^{8 \&}$ & $3.08 \pm 0.66^{\& \&}$ & $3.12 \pm 0.67^{\& \&}$ & $3.22 \pm 0.72^{\& \&}$ \\
\hline \multirow{4}{*}{$\begin{array}{l}\text { NAFLD } \\
\text { (female) }\end{array}$} & TC & $5.21 \pm 1.10 \%$ & $5.18 \pm 1.09 \%$ & $5.22 \pm 1.11^{\% \%}$ & $5.39 \pm 1.12 \%$ & $5.41 \pm 1.13 \%$ & $5.36 \pm 1.19 \%$ \\
\hline & $\mathrm{TG}$ & $2.04 \pm 1.13 \%$ & $3.16 \pm 1.44 \%$ & $3.98 \pm 1.97 \% \%$ & $4.11 \pm 2.54 \%$ & $3.92 \pm 2.56 \%$ & $2.83 \pm 2.47 \%$ \\
\hline & $\mathrm{HDL}-\mathrm{C}$ & $1.24 \pm 0.24 \%$ & $1.25 \pm 0.23 \%$ & $1.17 \pm 0.23^{\% \%}$ & $1.15 \pm 0.25 \%$ & $1.19 \pm 0.24 \%$ & $1.18 \pm 0.24 \%$ \\
\hline & LDL-C & $3.34 \pm 0.78 \%$ & $3.25 \pm 0.75 \%$ & $3.17 \pm 0.72^{\% \%}$ & $3.26 \pm 0.73 \%$ & $3.29 \pm 0.73 \%$ & $3.34 \pm 0.79 \%$ \\
\hline
\end{tabular}

Data are mean \pm standard deviation

Abbreviations: Con control group, NAFLD Nonalcoholic fatty liver disease, TC total cholesterol, $T G$ triglyceride, $H D L-C$ high-density lipoprotein-cholesterol, $L D L-C$ low-density lipoprotein-cholesterol

" $P<0.05$, compared with the control group. ${ }^{\# \#} P<0.01$, compared with the control group

\& $P<0.05$, compared with the male control group. ${ }^{\&} \&<<0.01$, compared with the male control group

${ }^{\%} P<0.05$, compared with the female control group. ${ }^{\%} P<0.01$, compared with the female control group 
TG $(\mathrm{mmol} / \mathrm{L})$

TG $(\mathrm{mmol} / \mathrm{L})$

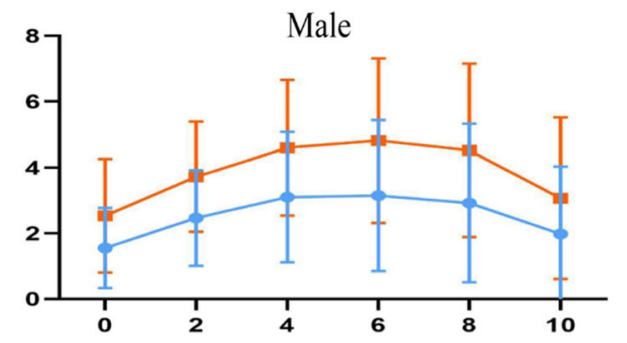

Time points during the OFTT

$\mathrm{B}$

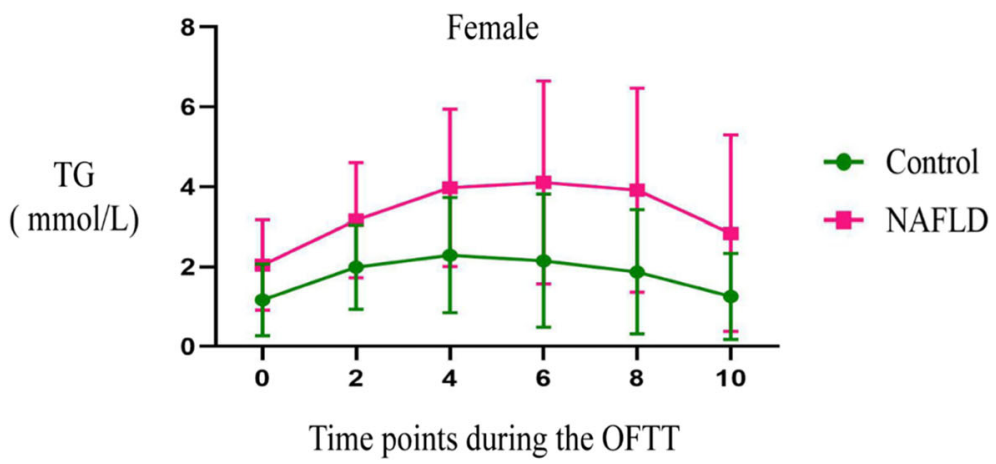

$\mathrm{C}$

Fig. 1 Triglyceride concentrations at time points during oral fat tolerance test; A-Total; B-Female; C-Male. TG-triglyceride; NAFLD- nonalcoholic fatty liver disease

\section{Factors associated with NAFLD}

The relationships of fasting BMI, WC, TC, TG, FBG, FINS, HOMA-IR, HDL-C, LDL-C, and fat load with NAFLD were determined using binary logistic regression analysis, and the factors that might predispose or protect the participants from NAFLD were further analyzed using forest plots. The parameters that were shown to be associated with the prevalence of NAFLD were BMI, WC, TC, TG, 2-h PTG (TG 2 h), 4-h PTG (TG 4h), 6-h PTG (TG $6 \mathrm{~h}$ ), HDL-C, LDL-C, HOMA-IR, FBG, and FINS (BMI: B 0.39, odds ratio [OR] 1.48, 95\% confidence interval [CI] 1.37-1.61; WC: B 0.13, OR 1.13, 95\% CI 1.10-1.16; TC: B 0.40, OR 1.50, 95\% CI 1.23-1.82; TG:
B 0.74, OR 2.09, 95\% CI 1.67-2.61; TG 2 h: B 0.68, OR 1.97, 95\% CI 1.65-2.35; TG 4 h: B 0.46, OR 1.59, 95\% CI 1.41-1.79; TG 6 h: B 0.37, OR 1.45, 95\% CI 1.31-1.60; HDL-C: B - 2.37, OR 0.09, 95\% CI 0.04-0.21; LDL-C: B 0.73, OR 2.07, 95\% CI 1.56-2.73; FBG: B 1.00, OR 2.72, 95\% CI 2.03-3.65; FINS: B 0.21, OR 1.23, 95\% CI 1.171.29; HOMA-IR: B 0.81, OR 2.24, 95\% CI 1.88-2.67; Table 5, Model 1, Fig. 2a).

After adjustment for age and sex, the factors listed above were still associated with NAFLD (BMI: B 0.40, OR 1.49, 95\% CI 1.37-1.62; WC: B, 0.15, OR 1.17, 95\% CI 1.13-1.21; TC: B 0.38, OR 1.46, 95\% CI 1.18-1.81; TG: B 0.69, OR 2.00, 95\% CI 1.60-2.49; TG 2 h: B 0.66, 
Table 4 Comparison of the characteristics of the NFTG and HFTG

\begin{tabular}{|c|c|c|c|c|}
\hline & Total & NFTG & HFTG & $P$ \\
\hline & $n=472$ & $n=322$ & $n=150$ & \\
\hline Age & $44 \pm 13$ & $43 \pm 14$ & $47 \pm 11$ & 0.176 \\
\hline Sex(male/female) & $224 / 248$ & $133 / 189$ & $91 / 59$ & 0.084 \\
\hline BMl & $25.93 \pm 4.05$ & $24.94 \pm 3.76$ & $28.06 \pm 3.85^{* *}$ & $<0.001$ \\
\hline FBG & $5.41(5.07,5.87)$ & $5.32(5.00,5.73)$ & $5.60(5.34,6.31)^{* *}$ & $<0.001$ \\
\hline FINS & $10.65(7.35,15.24)$ & $9.55(6.59,12.72)$ & $13.85(10.20,18.83)^{* *}$ & $<0.001$ \\
\hline HOMA-IR & $2.60(1.70,3.82)$ & $2.24(1.52,3.11)$ & $3.70(2.60,5.07)^{* *}$ & $<0.001$ \\
\hline $\mathrm{TC}$ & $4.72 \pm 1.01$ & $4.50 \pm 0.93$ & $5.19 \pm 1.02^{* *}$ & $<0.001$ \\
\hline $\mathrm{TG}$ & $1.66 \pm 1.31$ & $1.04 \pm 0.32$ & $2.98 \pm 1.62^{* *}$ & $<0.001$ \\
\hline $\mathrm{HDL}-\mathrm{C}$ & $1.25 \pm 0.28$ & $1.30 \pm 0.28$ & $1.14 \pm 0.24^{* *}$ & $<0.001$ \\
\hline LDL-C & $2.98 \pm 0.73$ & $2.82 \pm 0.69$ & $3.32 \pm 0.70^{* *}$ & $<0.001$ \\
\hline NAFLD & 155 (32.8\%) & 64 (19.9\%) & $91(60.7 \%)^{* *}$ & $<0.001$ \\
\hline Non-NAFLD & 317 (67.2\%) & $258(80.1 \%)$ & 59 (39.3\%) & $<0.001$ \\
\hline
\end{tabular}

Data are mean \pm standard deviation, median (interquartile range), or number (percentage)

Abbreviations NFTG normal fasting triglyceride group, HFTG High fasting triglyceride group, BMI body mass index, FBG fasting blood glucose, FINS fasting plasma insulin concentration, HOMA-IR homeostasis model assessment of insulin resistance, $T C$ total cholesterol, $T G$ triglyceride, $H D L-C$ high-density lipoproteincholesterol, $L D L-C$ low-density lipoprotein-cholesterol, NAFLD nonalcoholic fatty liver disease

${ }^{*} P<0.05$, compared with the NFT group. ${ }^{* *} P<0.01$, compared with the NFT group

OR 1.94, 95\% CI 1.62-2.32; TG 4 h: B 0.44, OR 1.55, 95\% CI 1.37-1.76; TG $6 \mathrm{~h}: \mathrm{B} 0.21$, OR 1.42, $95 \%$ CI 1.28-1.56; HDL-C: B - 2.51, OR 0.08, 95\% CI 0.03-0.20; LDL-C: B 0.69, OR 1.99, 95\% CI 1.47-2.69; FBG: B 0.94, OR 2.56, 95\% CI 1.88-3.47; FINS: B 0.22, OR 1.25, 95\% CI 1.19-1.31; HOMA-IR: B 0.82, OR 2.28, 95\% CI 1.902.72; Table 5, Model 2, Fig. 2b). To exclude the effects of age, sex and fasting TG, the data were further adjusted, after which high PTG was still associated with a higher likelihood of having NAFLD (TG $2 \mathrm{~h}$ : B 0.63, OR 1.88, 95\% CI 1.40-2.54; TG 4 h: B 0.32, OR 1.37, 95\% CI 1.14-1.65; TG 6 h: B 0.21, OR 1.23, 95\% CI 1.06-1.43; Table 5, Model 3, Fig. 2c).

\section{Characteristics of participants with differing levels of fat tolerance}

The participants were allocated to three groups according to their fasting TG and 4-h PTG concentrations. A 4-h PTG concentration $>2.5 \mathrm{mmol} / \mathrm{L}$ was defined as a high postprandial TG (HTG) concentration [21]. Participants with normal fasting triglyceride and PTG concentrations were placed in the normal fat tolerance group (NFT), those with a normal fasting concentration but an HTG were placed in the impaired fat tolerance (IFT) group, and those with a high fasting TG concentration were placed in the fasting hypertriglyceridemia (FHT) group (Table 6).

Furthermore, considering PTG peaked at $6 \mathrm{~h}$ after meal and 6-h PTG is a risk factor of NAFLD independent of age, sex and FTG, all the participants were allocated into different fat tolerance groups defined by FTG and 6-h PTG concentration (Table 7).
Although the criteria were different, across the three groups of different fat tolerance, fasting BMI, WC, FINS, HOMA-IR, TC, TG, and LDL-C all increased with decreasing fat tolerance, whereas HDL-C decreased with decreasing fat tolerance $(P<0.05)$. The age and FBG of the IFT and FHT groups were higher than those of the NFT group $(P<0.05)$, but there were no significant differences in age or FBG between the IFT and FHT groups. In addition, the incidence of NAFLD increased as the fat tolerance of the participants decreased. The incidence of NAFLD in the NFT group was relatively low, at $12.5 \%$, whereas $30.7 \%$ of the participants in the IFT group had NAFLD, and the incidence of NAFLD in the FHT group was much higher, at $60.6 \%$ (fat tolerance was defined according to FTG and 4-h PTG). The differences among the three groups were significant $(P<0.001)$. Meanwhile, across the three groups of different fat tolerance defined according to FTG and 6-h PTG, the incidences of NAFLD in NFT, IFT and FHT groups were $13.9,34.8$ and $60.6 \%$. The differences among the groups were significant at the same time $(P<0.001)$.

\section{Discussion}

According to the results of the present study, NAFLD is very common in people of Han ethnicity, with a prevalence of $32.8 \%$. By using binary logistic regression, BMI, WC, and TG were found to be associated with NAFLD, including TG after a fat load. After adjustment for age and sex, individuals with a high PTG remained more likely to have NAFLD.

By analogy with OGTT, the fasting TG and 4-h PTG concentrations during an OFTT were used as criteria to 
Table 5 Odds ratio of impacting factors for NAFLD

\begin{tabular}{|c|c|c|c|c|}
\hline Variables & Unstandardized Coefficient (B) & SE & Odds ratio $(95 \% \mathrm{Cl})$ & $P$ value \\
\hline \multicolumn{5}{|l|}{ Model 1} \\
\hline BMI & 0.39 & 0.04 & $1.48(1.37-1.61)$ & $<0.001$ \\
\hline WC & 0.13 & 0.01 & $1.13(1.10-1.16)$ & $<0.001$ \\
\hline TC & 0.40 & 0.10 & $1.50(1.23-1.82)$ & $<0.001$ \\
\hline TG & 0.74 & 0.11 & $2.09(1.67-2.61)$ & $<0.001$ \\
\hline TG $2 \mathrm{~h}$ & 0.68 & 0.09 & $1.97(1.65-2.35)$ & $<0.001$ \\
\hline TG $4 h$ & 0.46 & 0.06 & $1.59(1.41-1.79)$ & $<0.001$ \\
\hline TG $6 h$ & 0.37 & 0.05 & $1.45(1.31-1.60)$ & $<0.001$ \\
\hline $\mathrm{HDL}-\mathrm{C}$ & -2.37 & 0.42 & $0.09(0.04-0.21)$ & $<0.001$ \\
\hline LDL-C & 0.73 & 0.14 & $2.07(1.56-2.73)$ & $<0.001$ \\
\hline FBG & 1.00 & 0.15 & $2.72(2.03-3.65)$ & $<0.001$ \\
\hline FINS & 0.21 & 0.02 & $1.23(1.17-1.29)$ & $<0.001$ \\
\hline HOMA-IR & 0.81 & 0.09 & $2.24(1.88-2.67)$ & $<0.001$ \\
\hline \multicolumn{5}{|l|}{ Model 2} \\
\hline BMI & 0.40 & 0.04 & $1.49(1.37-1.62)$ & $<0.001$ \\
\hline WC & 0.15 & 0.02 & $1.17(1.13-1.21)$ & $<0.001$ \\
\hline TC & 0.38 & 0.11 & $1.46(1.18-1.81)$ & $<0.001$ \\
\hline TG & 0.69 & 0.11 & $2.00(1.60-2.49)$ & $<0.001$ \\
\hline TG $2 \mathrm{~h}$ & 0.66 & 0.09 & $1.94(1.62-2.32)$ & $<0.001$ \\
\hline TG $4 \mathrm{~h}$ & 0.44 & 0.06 & $1.55(1.37-1.76)$ & $<0.001$ \\
\hline TG $6 \mathrm{~h}$ & 0.21 & 0.05 & $1.42(1.28-1.56)$ & $<0.05$ \\
\hline $\mathrm{HDL}-\mathrm{C}$ & -2.51 & 0.46 & $0.08(0.03-0.20)$ & $<0.001$ \\
\hline LDL-C & 0.69 & 0.15 & $1.99(1.47-2.69)$ & $<0.001$ \\
\hline FBG & 0.94 & 0.16 & $2.56(1.88-3.47)$ & $<0.001$ \\
\hline FINS & 0.22 & 0.03 & $1.25(1.19-1.31)$ & $<0.001$ \\
\hline HOMA-IR & 0.82 & 0.09 & $2.28(1.90-2.72)$ & $<0.001$ \\
\hline \multicolumn{5}{|l|}{ Model 3} \\
\hline $\mathrm{TG} 2 \mathrm{~h}$ & 0.76 & 0.17 & $2.14(1.53-3.00)$ & $<0.001$ \\
\hline TG $4 \mathrm{~h}$ & 0.34 & 0.10 & $1.41(1.16-1.72)$ & $<0.05$ \\
\hline TG $6 \mathrm{~h}$ & 0.21 & 0.07 & $1.23(1.06-1.43)$ & $<0.05$ \\
\hline
\end{tabular}

Model 1-Crude OR; Model 2- Adjusted age and gender; Model 3- Adjusted age, gender fasting triglyceride and BMI

Abbreviations: BMI body mass index, WC waist circumference, FBG fasting blood glucose, FINS fasting plasma insulin concentration, HOMA-IR homeostasis model assessment of insulin resistance, $T C$ total cholesterol, $T G$ triglyceride, $T G 2 h$ triglyceride $2 \mathrm{~h}$ postprandially, $T G 4 h$ triglyceride $4 \mathrm{~h}$ postprandially, $H D L-C$ high-density lipoprotein-cholesterol, $L D L-C$ low-density lipoprotein-cholesterol, 95\% Cl 95\% confidence interval

define fat tolerance, and all the participants were allocated to three groups of differing fat tolerance, according to their fasting TG and PTG concentrations. According to the Greek consensus [21], PTG after the consumption of the high-fat meal during the OFTT of $>2.5 \mathrm{mmol} / \mathrm{L}$ was considered as high. Participants who had a fasting TG concentration within the normal range but a PTG higher than the desirable concentration were found to have a higher incidence of NAFLD than those with normal fasting TG and PTG concentrations. Therefore, if only the fasting TG concentration is measured as the normal clinical routine, a diagnosis of NAFLD may be missed. Instead, the measurement of PTG may help to identify patients in the early stages of NAFLD, which may lead to better clinical outcomes.

In this study, we analyzed basic characters such as BMI, WC and the incidences of NAFLD in different fat tolerance groups separately defined by FTG and 4-h PTG or FTG and 6-h PTG. The results showed that, the incidence of NAFLD climbed when fat tolerance declined. Although PTG peaked at $6 \mathrm{~h}$ after meal, 4-h PTG is more convenient for clinical practice. Furthermore, the use of a 4-h postprandial value to define hypertriglyceridemia is consistent with practices reported by multiple authors in other countries [22-25]. A study that assessed the determinants of PTG in healthy young 


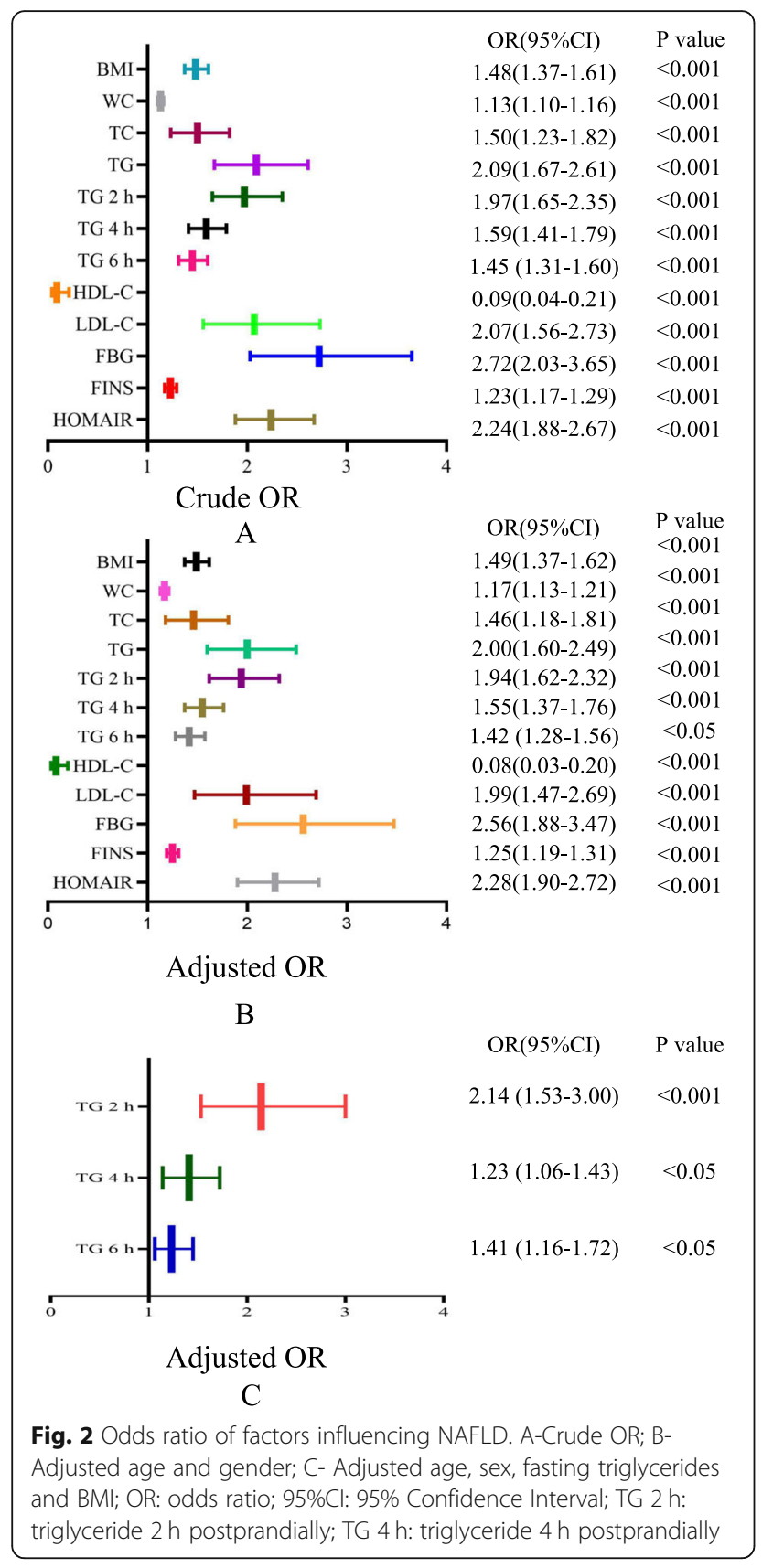

adults found a strong correlation between 4-h PTG and fasting TG, and therefore the authors came to the conclusion that 4-h PTG might be suitable as a replacement for fasting TG when this cannot easily be measured [26].

A meta-analysis conducted by Mihas et al. showed that a high TG concentration $4 \mathrm{~h}$ after a fat load is indicative of an excessive response [22]. The meta-analysis also suggested that a fat load of $70-79 \mathrm{~g}$ is optimal. However, the high-fat meal administered in the present study contained $100 \mathrm{~g}$ fat. Differences in the fat load may affect the postprandial lipid concentrations, as may the ethnicity of the participants. However, although the meta-
Table 6 Characteristics of different fat tolerance groups

\begin{tabular}{llll}
\hline & NFT $\boldsymbol{n}=\mathbf{1 9 2}$ & IFT $\boldsymbol{n}=\mathbf{1 3 0}$ & FHT $\boldsymbol{n}=\mathbf{1 5 0}$ \\
\hline Age & $40 \pm 13$ & $48 \pm 13^{\# \#}$ & $47 \pm 11^{\# \#}$ \\
Sex(male/female) & $66 / 126$ & $63 / 67$ & $91 / 59$ \\
BMI & $24.18 \pm 3.68$ & $26.07 \pm 3.60^{\# \#}$ & $28.06 \pm 3.85^{\# \#^{* *}}$ \\
WC & $82.43 \pm 10.96$ & $88.24 \pm 11.32^{\# \#}$ & $94.71 \pm 9.79^{\# \# *}$ \\
FBG & $5.32 \pm 0.56$ & $5.72 \pm 1.36^{\#}$ & $6.00 \pm 1.29^{\# \#}$ \\
FINS & $9.56 \pm 5.20$ & $12.18 \pm 6.95^{\#}$ & $15.95 \pm 9.91^{\# \#^{*}}$ \\
HOMA-IR & $2.29 \pm 1.36$ & $3.22 \pm 2.51^{\# \#}$ & $4.33 \pm 2.96^{\# \#^{*}}$ \\
TC & $4.34 \pm 0.88$ & $4.74 \pm 0.95^{\# \#}$ & $5.19 \pm 1.02^{\# \#^{* *}}$ \\
TG & $0.88 \pm 0.26$ & $1.28 \pm 0.25^{\# \#}$ & $2.98 \pm 1.62^{\# \#^{* *}}$ \\
HDL-C & $1.34 \pm 0.29$ & $1.23 \pm 0.26^{\# \#}$ & $1.14 \pm 0.24^{\# \#^{*}}$ \\
LDL-C & $2.66 \pm 0.65$ & $3.05 \pm 0.69^{\# \#}$ & $3.32 \pm 0.70^{\# \#^{* *}}$ \\
NAFLD & $24(12.5 \%)$ & $40(30.7 \%)$ & $91(60.6 \%))^{\# \# \#^{* *}}$
\end{tabular}

Data are mean \pm standard deviation, median (interquartile range), or number (percentage)

Abbreviations: $B M I$ body mass index, WC waist circumference, $F B G$ fasting blood glucose, FINS fasting plasma insulin concentration, HOMA-IR

homeostasis model assessment of insulin resistance, TC total cholesterol, TG triglyceride, HDL-C high-density lipoprotein-cholesterol, LDL-C low-density lipoprotein-cholesterol, NAFLD nonalcoholic fatty liver disease, NFT normal fat tolerance, IFT impaired fat tolerance, $F H T$ fasting hypertriglyceridemia ${ }^{\#} P<0.05$, compared with the NFT group. ${ }^{\#} P<0.01$, compared with the NFT group. ${ }^{*} P<0.05$, compared with the IFT group. ${ }^{* *} P<0.01$, compared with the IFT group

analysis was only of studies of Caucasians and the participants in the present study were all Chinese, their TG concentrations also peaked at $4 \mathrm{~h}$ postprandially. To determine the most suitable time point for the assessment of postprandial lipids during an OFTT in Chinese people, further studies are required. In addition, the definition of PTG remains unclear. The criteria used in the

Table 7 Characteristics of different fat tolerance groups allocated according to FTG and $6 \mathrm{~h}$ PTG

\begin{tabular}{llll}
\hline & NFT & IFT & FHT \\
& $\boldsymbol{n}=\mathbf{2 3 0}$ & $\boldsymbol{n}=\mathbf{9 2}$ & $\boldsymbol{n}=\mathbf{1 5 0}$ \\
\hline Age & $41 \pm 14$ & $49 \pm 12^{\# \#}$ & $47 \pm 11^{\# \#}$ \\
Sex(male/female) & $82 / 148$ & $51 / 41$ & $91 / 59$ \\
BMl & $24.4 \pm 3.67$ & $26.29 \pm 3.65^{\# \#}$ & $28.06 \pm 3.85^{\# \# *}$ \\
WC & $82.7 \pm 11.52$ & $89.96 \pm 9.52^{\# \#}$ & $94.71 \pm 9.79^{\# \#}$ \\
FBG & $5.36 \pm 0.75$ & $5.77 \pm 1.38^{\#}$ & $6.00 \pm 1.29^{\# \#}$ \\
FINS & $10.12 \pm 5.7$ & $11.86 \pm 6.87^{\#}$ & $15.95 \pm 9.91^{\# \#{ }^{* *}}$ \\
HOMA-IR & $2.46 \pm 1.62$ & $3.15 \pm 2.56^{\#}$ & $4.33 \pm 2.96^{\# \# \#^{* *}}$ \\
TC & $4.38 \pm 0.86$ & $4.81 \pm 1.01^{\#}$ & $5.19 \pm 1.02^{\# \# *}$ \\
TG & $0.95 \pm 0.29$ & $1.28 \pm 0.27^{\# \#}$ & $2.98 \pm 1.62^{\# \# \#^{* *}}$ \\
HDL-C & $1.33 \pm 0.28$ & $1.22 \pm 0.26^{\#}$ & $1.14 \pm 0.24^{\# \#}$ \\
LDL-C & $2.69 \pm 0.63$ & $3.13 \pm 0.73^{\# \#}$ & $3.32 \pm 0.7^{\# \#}$ \\
NAFLD & $32(13.9 \%)$ & $32(34.8 \%)^{\# \#}$ & $91(60.6 \%)^{\# \# *}$ \\
\hline Data are mean &
\end{tabular}

Data are mean \pm standard deviation, or number (percentage)

\# $P<0.05$, compared with the NFT group. ${ }^{\# \#} P<0.01$, compared with the NFT group. ${ }^{*} P<0.05$, compared with the IFT group. ${ }^{* *} P<0.01$, compared with the IFT group 
present study was higher than that after a daily meal recommended by the EAS consensus statement [16] and that after a daily Chinese breakfast in overweight people [21] while lower than that in another Mexican study [27]. Disparities in the levels used in the various studies may be related to the differing ethnicity of the participants and differences in their standard dietary calorie and fat intake.

Participants with NAFLD were found to have higher BMI, WC, SBP, DBP, and HOMA-IR, which is consistent with previous studies. Obese but metabolically healthy individuals have been shown to be at a greater risk of NAFLD progression than metabolically healthy individuals of a normal weight [28]. In addition, WC is a risk factor for NAFLD because it is associated with IR and hypertension [29-31]. An analysis of the characteristics of participants with differing fasting TG concentrations showed that BMI is higher in individuals with high fasting TG concentrations. Furthermore, the study by Nogueira et al. showed that overweight and obesity are associated with a high prevalence of dyslipidemia [32], and another study showed that obese and insufficiently active male adolescents are more likely to have a high circulating TG concentration [33].

High TC, TG, and LDL-C concentrations are risk factors for CVD [34], whereas HDL-C has protective effects [35]. HDL-C is mainly involved in the reverse transport of cholesterol from extrahepatic tissues to the liver, but it can also inhibit the uptake of LDL-C by arterial smooth muscle cells and prevent the accumulation of cholesterol in cells. Therefore, HDL-C has antiatherosclerotic effects and is often used as a marker of CVD risk [36]. In the present study, patients with NAFL $\mathrm{D}$ were found to have abnormal lipid metabolism, indicated by high concentrations of TC, TG, and LDL-C and a low concentration of HDL-C. ApoB regulates the secretion of very low-density lipoprotein, which is present at high circulating concentrations in patients with obesity, T2DM, and hypertriglyceridemia. Therefore, the high ApoB concentration in the NAFLD group is further evidence of dyslipidemia in these patients.

The TG concentration peaked later after a meal in the NAFLD group, which may imply that the meal-induced change in serum TG is larger in patients with NAFLD than in normal individuals. An impairment in TG metabolism is also suggested by the long period of time taken for the TG concentration to return to baseline following a high-fat meal.

Dyslipidemia is the principal etiologic factor in the development of NAFLD in non-diabetic patients [37]. A previous study showed that dyslipidemia is common in lean non-diabetic patients with NAFLD, with similar lipid profiles being identified in such patients to those who were overweight or obese [38]. The results of this study also suggested that high TG and low HDL-C concentrations may influence the development of NAFLD in non-diabetic patients.

\section{Study strength and limitations}

The strengths of the present study are as follows. To the best of our knowledge, this was the first study to use a standardized OFTT in a large sample of Chinese patients to assess the relationship between PTG and NAFLD. In this study, 4-h PTG is significantly related to NAFLD. The 4-h time point after eating is consistent with the results of a previous study that showed a strong correlation between 4-h PTG and fasting TG [26].

Some limitations to the study should also be acknowledged. Abdominal ultrasound is not sensitive to diagnose fatty liver. Subjects with mild steatosis are possibly missed. Therefore, the conclusion of this study may be limited to some extent. The results of the present study cannot be extrapolated to other populations because it only included Chinese patients. In a clinical trial, it is difficult to match participants for their age and sex. However, analysis of the data for each sex showed that patients with NAFLD of both sexes have poorer fasting lipid profiles than those without. Furthermore, after adjustment for age and sex, 4-h PTG remained positively associated with a higher likelihood of having NAFLD.

\section{Conclusions}

A high 4-h PTG concentration were found to be significantly and positively related to NAFLD. In addition, individuals with a high PTG have a higher prevalence of NAFLD. Thus, 4-h PTG may represent a potential marker of NAFLD. The results showed that the incidence of NAFLD in individuals with normal fasting TG but high PTG is high, at $30.7 \%$. Therefore, the measurement of fasting TG alone may result in the misdiagnosis of some metabolically unhealthy patients who have normal fasting TG concentrations. NAFLD is asymptomatic in most patients, and therefore it remains a diagnosis of exclusion. Although liver biopsy is the gold-standard method of distinguishing the different stages of NAFLD, it is not always used in clinical practice because of its invasiveness. Therefore, early identification and treatment of NAFLD may become more feasible if PTG is measured. In addition, nutritional factors influence NAFLD, and PTG may be useful as a means of monitoring the efficacy of therapeutic interventions in patients with NAFLD.

\footnotetext{
Abbreviations

NAFLD: Nonalcoholic fatty liver disease; NASH: Nonalcoholic steatohepatitis; CVD: Chronic cardiovascular disease; BMI: Body mass index; SBP: Systolic blood pressure; DBP: Diastolic blood pressure; WC: Waist circumference; FBG: Fasting blood glucose; FINS: Fasting plasma insulin concentration; HOMA-IR: Homeostasis model assessment of insulin resistance; TC: Total cholesterol; TG: Triglyceride; HDL-C: High-density lipoprotein-cholesterol;
} 
LDL-C: Low-density lipoprotein-cholesterol; ApoA1: Apolipoprotein A1; ApoB: Apolipoprotein B; Con: Control group; NFTG: Normal fasting triglyceride group; HFTG: High fasting triglyceride group; NFT: Normal fat tolerance; IFT: Impaired fat tolerance; FHT: Fasting hypertriglyceridemia; TG 2 h: 2-h postprandial triglyceride; TG 4 h: 4-h postprandial triglyceride; $95 \%$ Cl: $95 \%$ confidence interval

\section{Supplementary Information}

The online version contains supplementary material available at https://doi. org/10.1186/s12944-021-01483-z.

Additional file 1: Table S1. Oral glucose tolerance test results of participants with or without NAFLD

\section{Acknowledgments}

We sincerely thank the teachers of the Clinical Medical Research Centre of Hebei General Hospital for their help with the study. We also thank Mark Cleasby, PhD, from Liwen Bianji, Edanz Group China (www.liwenbianji.cn/ac), for editing the English text of a draft of this manuscript.

\section{Authors' contributions}

Xiaoyu Hou: Formal analysis, Writing-Original draft; Yunpeng Guan: Formal analysis, visualization; An Song, Jiajun Zhao, Shuchun Chen, Limin Wei and Huijuan Ma: Conceptualization, Supervision; Yong Tang, Luping Ren: Methodology, Supervision, Writing-Reviewing; Guangyao Song: Conceptualization, Methodology, Supervision, Project administration, Writing-Reviewing and Editing. Xiaoyu Hou and Yunpeng Guan contributed equally to this manuscript. The authors read and approved the final manuscript.

\section{Funding}

This study was funded by the Research Fund of Hebei Provincial Government.

\section{Availability of data and materials}

The datasets generated in this study and the protocol are available on reasonable request. Please contact the corresponding author.

\section{Declarations}

\section{Ethics approval and consent to participate}

The study complied with the principles of the Declaration of Helsinki, the protocol was approved by the ethics committee of Hebei General Hospital, and all the participants gave their written informed consent.

\section{Consent for publication}

Not applicable.

\section{Competing interests}

The authors declare no conflicts of interests.

\section{Author details}

${ }^{1}$ Department of Internal Medicine, Hebei Medical University, Shijiazhuang, Hebei, China. ${ }^{2}$ Department of Endocrinology, Hebei General Hospital, 348, Heping West Road, Shijiazhuang, Hebei 050051, People's Republic of China. ${ }^{3}$ Key laboratory of Endocrinology, Ministry of Health, Department of Endocrinology, Peking Union Medical College Hospital, Peking Union Medical College, Chinese Academy of Medical Sciences, Beijing, China. ${ }^{4}$ Department of Endocrinology and Metabolism, Shandong Provincial Hospital Affiliated to Shandong University, Jinan, Shandong, China.

Received: 15 February 2021 Accepted: 18 May 2021

Published online: 25 May 2021

\section{References}

1. Fazel Y, Koenig AB, Sayiner M, Goodman ZD, Younossi ZM. Epidemiology and natural history of non-alcoholic fatty liver disease. Metabolism. 2016; 65(8):1017-25. https://doi.org/10.1016/j.metabol.2016.01.012.

2. Younossi ZM, Koenig AB, Abdelatif D, Fazel Y, Henry L, Wymer M. Global epidemiology of nonalcoholic fatty liver disease-meta-analytic assessment of prevalence, incid ence, and outcomes. Hepatology. 2016;64(1):73-84. https://doi.org/10.1002/hep.28431.

3. Noureddin M, Vipani A, Bresee C, Todo T, Kim IK, Alkhouri N, et al. NASH leading cause of liver transplant in women: updated analysis of indications for liver transplant and ethnic and gender variances. Am J Gastroenterol. 2018;113(11):1649-59. https://doi.org/10.1038/s41395-018-0088-6.

4. Younes R, Bugianesi E. Should we undertake surveillance for HCC in patients with NAFLD? J Hepatol. 2018;68(2):326-34. https://doi.org/10.1016/j.jhep.201 7.10.006.

5. Stine JG, Wentworth BJ, Zimmet A, Rinella ME, Loomba R, Caldwell SH, et al. Systematic review with meta-analysis: risk of hepatocellular carcinoma in non-alcoholic steatohepatit is without cirrhosis compared to other liver diseases. Aliment Pharmacol Ther. 2018;48(7):696-703. https://doi.org/1 0.1111/apt.14937.

6. Buzzetti E, Pinzani M, Tsochatzis EA. The multiple-hit pathogenesis of nonalcoholic fatty liver disease (NAFLD). Metabolism. 2016;65(8):1038-48. https://doi.org/10.1016/j.metabol.2015.12.012.

7. Alshamiri M, Ghanaim MMA, Barter P, Chang KC, Li JJ, Matawaran BJ, et al. Expert opinion on the applicability of dyslipidemia guidelines in Asia and the Middle East. Int J Gen Med. 2018;11:313-22. https://doi.org/10.2147/ JJGM.S160555.

8. Loria P, Marchesini G, Nascimbeni F, Ballestri S, Maurantonio M, Carubbi F, et al. Cardiovascular risk, lipidemic phenotype and steatosis. A comparative analysis of cirrhotic and non-c irrhotic liver disease due to varying etiology. Atherosclerosis. 2014;232(1):99-109. https://doi.org/10.1016/j.a therosclerosis.2013.10.030.

9. Lonardo A, Loria P, Leonardi F, Borsatti A, Neri P, Pulvirenti M, et al. Fasting insulin and uric acid levels but not indices of iron metabolism are independent predictors of non-alcoholic fatty liver disease. A case-control study. Dig Liver Dis. 2002;34(3):204-11. https://doi.org/10.1016/S15908658(02)80194-3.

10. Hsiao PJ, Kuo KK, Shin SJ, Yang YH, Lin WY, Yang JF, et al. Significant correlations between severe fatty liver and risk factors for metabolic syndrome. J Gastroenterol Hepatol. 2007;22(12):2118-23. https://doi.org/1 0.1111/j.1440-1746.2006.04698.x.

11. Tutunchi $H$, Saghafi-Asl M, Asghari-Jafarabadi M, Ostadrahimi A. The relationship between severity of liver steatosis and metabolic parameters in a sample of Iranian adults. BMC Res Notes. 2020;13(1):218. https://doi.org/1 0.1186/s13104-020-05059-5.

12. Schönknecht $Y B$, Crommen S, Stoffel-Wagner B, Coenen M, Fimmers R, Holst JJ, et al. Acute effects of three different meal patterns on postprandial metabolism in older individuals with a risk phenotype for Cardiometabolic diseases: a randomized controlled crossover trial. Mol Nutr Food Res. 2020; 64:e1901035.

13. Petersen KS, Rogers CJ, West SG, Proctor DN, Kris-Etherton PM. The effect of culinary doses of spices in a high-saturated fat, high-carbohydrate meal on postprandia I lipemia and endothelial function: a randomized, controlled, crossover pilot trial. Food Funct. 2020;11(4):3191-200. https://doi.org/10.103 9/C9FO02438G

14. Mach F, Baigent C, Catapano AL, Koskinas KC, Casula M, Badimon L, et al. 2019 ESC/EAS guidelines for the management of dyslipidaemias: lipid modification to reduce cardiovasc ular risk. Eur Heart J. 2020;41:111-88.

15. Cosentino F, Grant PJ, Aboyans V, Bailey CJ, Ceriello A, Delgado V, et al. 2019 ESC guidelines on diabetes, pre-diabetes, and cardiovascular diseases developed in collaboration with the EASD. Eur Heart J. 2020;41:255-323.

16. Nordestgaard BG, Langsted A, Mora S, Kolovou G, Baum H, Bruckert E, et al. Fasting is not routinely required for determination of a lipid profile: clinical and laboratory implications including flagging at desirable concentration cut-points-a joint consensus statement from th e European atherosclerosis society and European Federation of Clinical Chemistry and Laboratory Medicine. Eur Heart J. 2016;37(25):1944-58. https://doi.org/10.1093/eurhea rtj/ehw152.

17. Kolovou GD, Watts GF, Mikhailidis DP, Mora S, Bilianou H, Panotopoulos G, et al. Postprandial Hypertriglyceridaemia revisited in the era of non-fasting lipid profiles: executive summary of a 2019 expert panel statement. Curr Vasc Pharmacol. 2019;17:538-40.

18. Quon MJ. Limitations of the fasting glucose to insulin ratio as an index of insulin sensitivity. J Clin Endocrinol Metab. 2001;86(10):4615-7. https://doi. org/10.1210/jcem.86.10.7952.

19. National Workshop on Fatty L, Alcoholic Liver Disease CSoHCMA, Fatty Liver Expert Committee CMDA. Guidelines of prevention and treatment for 
nonalcoholic fatty liver disease: a 2018 update. Zhonghua Gan Zang Bing Za Zhi. 2018;26:195-203.

20. Ratziu V, Bellentani S, Cortez-Pinto H, Day C, Marchesini G. A position statement on NAFLD/NASH based on the EASL 2009 special conference. J Hepatol. 2010;53(2):372-84. https://doi.org/10.1016/j.jhep.2010.04.008.

21. Tian F, Xiang QY, Zhang MY, Chen YQ, Lin QZ, Wen T, et al. Changes in non-fasting concentrations of blood lipids after a daily Chinese breakfast in overweight s ubjects without fasting hypertriglyceridemia. Clin Chim Acta. 2019;490:147-53. https://doi.org/10.1016/j.cca.2019.01.004.

22. Mihas C, Kolovou GD, Mikhailidis DP, Kovar J, Lairon D, Nordestgaard BG, et al. Diagnostic value of postprandial triglyceride testing in healthy subjects: a meta-analysis. Curr Vasc Pharmacol. 2011;9(3):271-80. https://doi. org/10.2174/157016111795495530.

23. Gavra P, Melidonis A, Iraklianou S, Mihas C, Kolovou V, Mavrogeni S, et al. Alterations in plasma triglyceride concentrations following two Oral meals with different fat content in patients with type 2 diabetes mellitus. Curr Vasc Pharmacol. 2018;16(4):385-92. https://doi.org/10.2174/15701611156661 70529084621.

24. Tentolouris N, Kanellos PT, Siami E, Athanasopoulou E, Chaviaras N, Kolovou $G$, et al. Assessment of the validity and reproducibility of a novel standardized test meal for the study of Pos tprandial triacylglycerol concentrations. Lipids. 2017;52(8):675-86. https://doi.org/10.1007/s11745-01 7-4275-9.

25. Perez-Martinez P, Alcala-Diaz JF, Kabagambe EK, Garcia-Rios A, Tsai MY, Delgado-Lista J, et al. Assessment of postprandial triglycerides in clinical practice: validation in a general population and coronary heart disease patients. J Clin Lipidol. 2016;10(5):1163-71. https://doi.org/10.1016/j.jacl.2016. 05.009.

26. Tarigan TJE, Khumaedi Al, Wafa S, Johan M, Abdullah M, Surono IS, et al. Determinant of postprandial triglyceride levels in healthy young adults. Diabetes Metab Syndr. 2019;13(3):1917-21. https://doi.org/10.1016/j.dsx.201 9.04.027.

27. Sevilla-González MDR, Aguilar-Salinas CA, Muñóz-Hernández L, AlmedaValdés $P$, Mehta $R$, Zubirán $R$, et al. Identification of a threshold to discriminate fasting hypertriglyceridemia with postprandial values. Lipids Health Dis. 2018;17(1):156. https://doi.org/10.1186/s12944-018-0803-8.

28. Lonardo A, Mantovani A, Lugari S, Targher G. Epidemiology and pathophysiology of the association between NAFLD and metabolically healthy or metabo lically unhealthy obesity. Ann Hepatol. 2020;19(4):359-66. https://doi.org/10.1016/j.aohep.2020.03.001.

29. Bedogni G, Bellentani S, Miglioli L, Masutti F, Passalacqua M, Castiglione A, et al. The fatty liver index: a simple and accurate predictor of hepatic steatosis in the general population. BMC Gastroenterol. 2006;6(1):33. https:// doi.org/10.1186/1471-230X-6-33.

30. Zhao YC, Zhao GJ, Chen Z, She ZG, Cai J, Li H. Nonalcoholic fatty liver disease: an emerging driver of hypertension. Hypertension. 2020;75:275-84.

31. Pan X, Xie X, Peng H, Cai X, Li H, Hong Q, et al. Risk prediction for nonalcoholic fatty liver disease based on biochemical and dietary variables in a Chinese Han population. Front Public Health. 2020;8:220. https://doi.org/1 0.3389/fpubh.2020.00220.

32. Nogueira-de-Almeida CA, Mello ED. Correlation of body mass index Z-scores with glucose and lipid profiles among overweight and obese ch ildren and adolescents. J Pediatr. 2018;94(3):308-12. https://doi.org/10.1016/j.jped.2017. 06.012.

33. Dejavitte RAS, Enes CC, Nucci LB. Prevalence of metabolic syndrome and its associated factors in overweight and obese adolescents. J Pediatr Endocrinol Metab. 2020;33(2):233-9. https://doi.org/10.1515/jpem-2019-03 69.

34. Kauffman SAE, Averill MM, Delaney JAC, Lemaitre RN, Howard BV, Fretts AM. Associations of diet quality and blood serum lipoprotein levels in a population at high risk for diab etes: the strong heart family study. Eur J Clin Nutr. 2020;74(7):1084-90. https://doi.org/10.1038/s41430-019-0539-1.

35. Lamprea-Montealegre JA, McClelland RL, Otvos JD, Mora S, Koch M, Jensen MK, et al. Association of High-Density Lipoprotein Particles and HighDensity Lipoprotein Apolipoprotein C-III C ontent with cardiovascular disease risk according to kidney function: the multi-ethnic study of Athe rosclerosis. J Am Heart Assoc. 2019;8:e013713.

36. Lüscher TF, Landmesser U, von Eckardstein A, Fogelman AM. High-density lipoprotein: vascular protective effects, dysfunction, and potential as therapeutic target. Circ Res. 2014;114(1):171-82. https://doi.org/10.1161/ CIRCRESAHA.114.300935.
37. Ray $G$, Agarwala $T$. A study of metabolic parameters in non diabetic patients with non alcoholic fatty liver disease - importance of Dyslipidemia. Arq Gastroenterol. 2019;56(3):270-5. https://doi.org/10.1590/s0004-2803.201 900000-51.

38. Kumar R, Rastogi A, Sharma MK, Bhatia V, Garg H, Bihari C, et al. Clinicopathological characteristics and metabolic profiles of non-alcoholic fatty liver disease in Indian patients with normal body mass index: do they differ from obese or overweight non-alcoholic fat ty liver disease? Indian J Endocrinol Metab. 2013;17(4):665-71. https://doi.org/10.4103/2230-8210.113 758.

\section{Publisher's Note}

Springer Nature remains neutral with regard to jurisdictional claims in published maps and institutional affiliations.
Ready to submit your research? Choose BMC and benefit from:

- fast, convenient online submission

- thorough peer review by experienced researchers in your field

- rapid publication on acceptance

- support for research data, including large and complex data types

- gold Open Access which fosters wider collaboration and increased citations

- maximum visibility for your research: over $100 \mathrm{M}$ website views per year

At BMC, research is always in progress.

Learn more biomedcentral.com/submissions 